\title{
An unusual cause of central cyanosis in a patient with rheumatic heart disease
}

\author{
PK LEE, SY SO, JSF CHOW \\ From the Department of Medicine, University of Hong Kong
}

\begin{abstract}
Rheumatic valvular heart disease, especially mitral stenosis, is commonly associated with abnormal pulmonary function. Systemic arterial oxygen saturation in such patients, however, almost always stays within the normal range or is only minimally reduced, ${ }^{1}$ except perhaps during spells of acute pulmonary oedema. We report a case of "silent" pulmonary arteriovenous fistula occurring in a patient with aortic and mitral stenosis. The only clues to the diagnosis before cardiac catheterisation were the presence of central cyanosis and a suspicious calcified hilar shadow.
\end{abstract}

\section{Case report}

A 56 year old woman with a long history of rheumatic heart disease was referred for assessment because of progressive deterioration in exercise capacity and recurrent ankle oedema. She was a non-smoker and gave no history suggestive of chronic respiratory disease. Physical examination showed central cyanosis but no finger clubbing. Mild bilateral ankle oedema was present. There was no cutaneous or mucosal telangiectasia. Jugular venous pressure was not raised. The carotid pulse was of small volume and slow rising in character. A sustained apical impulse was palpable at the 5 th intercostal space, $1 \mathrm{~cm}$ lateral to the midclavicular line. The first heart sound was normal and the aortic component of the second heart sound was diminished. A harsh systolic murmur was heard over both sternal borders with radiation to the neck. A soft mid diastolic murmur was detected at the cardiac apex. Examination of the respiratory system showed no abnormality.

A resting electrocardiogram showed atrial fibrillation. A chest radiograph showed a normal cardiothoracic ratio and mild upper lobe venous diversion. A calcific, lobulated mass was seen over the right hilar region (fig 1). There was no evidence of calcification over the mitral or aortic valve areas. Two dimensional echocardiography showed features of tight aortic and mitral stenosis. Spirometry showed the FEV to be $1.61(86 \%$ of predicted normal) and the forced vital capacity (FVC) to be 1.81 ( $75 \%$ of predicted normal), giving an FEV $1 /$ FVC ratio of 0.87 .

At cardiac catheterisation the aortic oxygen saturation was found to be only $82 \%$; pulmonary arterial saturation was $51 \%$. The cardiac output calculated by the Fick principle was $3.11 / \mathrm{min}$. Retrograde catheterisation of the left ventricle through the stenotic aortic valve was unsuccessful. A very narrow aortic orifice was seen on the aortogram. Pulmonary arterial pressure was $32 / 20 \mathrm{~mm} \mathrm{Hg}$, with a mean

Address for reprint requests: Dr PK Lee, Department of Medicine, Hong Kong University, Queen Mary Hospital, Pokfulam Road, Hong Kong.

Accepted 12 April 1985

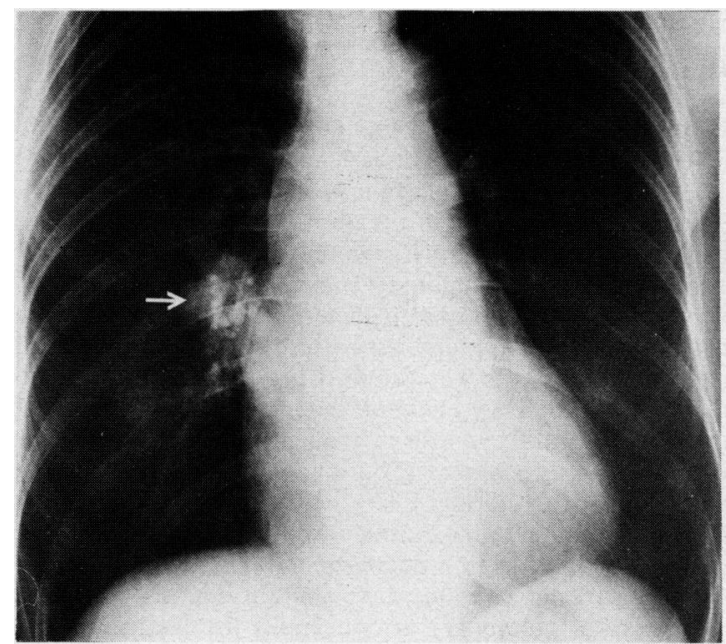

Fig 1 Chest radiograph showing a calcific right hilar mass (white arrow).

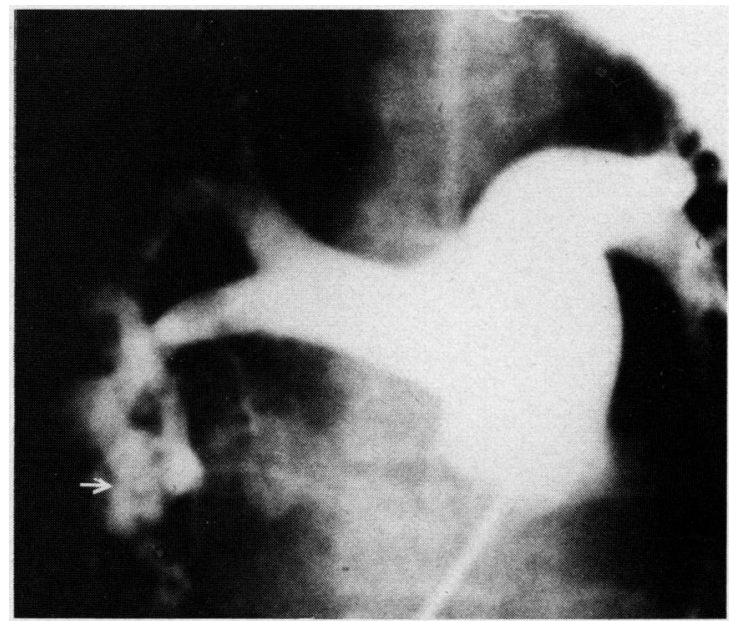

Fig 2 Pulmonary arteriogram showing tortuous vascular channels of the arteriovenous fistula (white arrow).

pulmonary arterial wedge pressure of $15 \mathrm{~mm} \mathrm{Hg}$. A pulmonary arteriogram showed a large, tortuous fistula arising from the branches of the right upper lobe and right middle lobe arteries and draining through a tangle of distended, tortuous vascular channels into the left atrium (fig 2). 
During operation through a split sternum approach, the pulmonary arteriovenous fistula was found to go from the basal portion of the anterior segment of the right upper lobe to the adjoining right middle lobe. The fistula was resected together with a wedge of lung tissue. The patient was then put on cardiopulmonary bypass. The stenotic aortic and mitral valves were replaced by Björk-Shiley prostheses. The postoperative course was eventful and she was well when discharged from hospital taking anticoagulant and digitalis.

\section{Discussion}

Peripheral cyanosis is not uncommon among patients with severe rheumatic valvular disease and a low cardiac output. The occurrence of central cyanosis in these patients is, however, distinctly unusual in the absence of overt pulmonary oedema. The referring physician initially attributed this patient's cyanosis to pulmonary congestion secondary to her valvular lesions. At cardiac catheterisation, however, only a marginal increase in pulmonary artery and pulmonary arterial wedge pressures was found. Chronic obstructive lung diseases are relatively common among patients of this age group, and central cyanosis is frequently encountered in the late stages of these diseases; but the mild reduction of FVC and $\mathrm{FEV}_{1}$ with a normal $\mathrm{FEV}_{1} / \mathrm{FVC}$ ratio excluded such a respiratory cause for the central cyanosis in our patient. This pattern of lung function derangement is in fact quite common for patients with appreciable mitral stenosis.

Congenital pulmonary arteriovenous fistulas commonly present in adult life as incidental chest radiographic findings or recurrent bleeding from mucosal telangiectases. Dyspnoea and cyanosis are found only among patients with large shunts. ${ }^{2}$ In these cases continuous or systolic murmum are frequently detected. The present patient, as in two other reported cases with coexistent cardiac valvular lesions, ${ }^{34}$ d not have a thoracic murmur that could be attributed to a shunt through the fistula. Although a small percentage of pulmonary arteriovenous fistulas may have no audible muf mur, the murmur produced by the shunt in this patient $\frac{0}{75}$ more likely to have been masked by the accompanying cal diac murmurs. The perihilar location and calcification of the fistula made the initial interpretation of the chest radiograph more puzzling. Although both features have been described in previous reports on pulmonary arteriovenous fistula, they are exceptional. ${ }^{5}$ Indeed the shadows were thought to repres sent calcific hilar lymph glands before the fistula was confirmed by arteriogram.

The case illustrates an unusual combination of congenit and acquired cardiac lesions occurring in an adult patient. Furthermore, it emphasises the fact that central cyanosis rarely causally related to rheumatic valvular disease. The coexistence warrants thorough investigation for a second pathological condition.

\section{References}

1 Dawson A. Pulmonary effects of mitral stenosis. Practical Cardit ology 1981;7:10:55-61.

2 Foley RE, Boyd DP. Pulmonary arteriovenous aneurysm. SuPg Clin N Am 1961;41:801-6.

3 Beard CW, Roberts WC, Kahler RL. Pulmonary arterio-veno@s fistula and rheumatic heart disease. Am Heart J 1966;71:390-

4 Steinberg I. Pulmonary arterio-venous fistulas of the medial ba్ㅐㅇ segment of the right lower lobe: a note on absence of vascupirr bruit. Dis Chest 1958;33:86-92.

5 Seaman W, Goldman A. Roentgen aspects of pulmonary arterß̈ venous fistula. Arch Intern Med 1952;89:70-81. 